\title{
Downregulation of heparanase by RNA interference inhibits invasion and tumorigenesis of hepatocellular cancer cells in vitro and in vivo
}

\author{
ZHEN XIONG $^{1,2}$, MU-HAN LÜ ${ }^{1}$, YA-HAN FAN ${ }^{1}$, YA-LING CAO ${ }^{1}$, CHANG-JIANG HU $^{1}$, YU-YUN WU ${ }^{1}$, \\ SHU-MIN WANG ${ }^{1}$, GANG LUO $^{1}$, DIAN-CHUN FANG ${ }^{1}$, CHUAN LI $^{1}$ and SHI-MING YANG ${ }^{1}$ \\ ${ }^{1}$ Institute of Gastroenterology, Southwest Hospital, Third Military Medical University, Chongqing 400038; \\ ${ }^{2}$ Department of Gastroenterology, the 181st Hospital of Chinese People Liberation Army, Guiling, \\ Guangxi Zhuang Autonomous Region 541002, P.R. China
}

Received October 10, 2011; Accepted December 12, 2011

DOI: 10.3892/ijo.2012.1338

\begin{abstract}
Heparanase is an endoglycosidase that degrades heparan sulfate, the main polysaccharide constituent of the extracellular matrix and basement membrane. The expression of heparanase is associated with invasion, as well as the angiogenic and metastatic potential of diverse malignant tumors. We used RNA interference strategies to evaluate the role of human heparanase in a liver cancer cell line and to explore the therapeutic potential of its specific targeting. Using an online siRNA tool, we designed three small interfering RNA sequences to target the heparanase coding region and cloned them into the pGenesil-1 vector. The siRNA vectors were transfected into HepG2 liver cancer cells. Heparanase expression was measured by real-time RT-PCR and Western blotting. Cell proliferation was detected by MTT staining and plate colony formation. Cell cycle analysis was performed by flow cytometry. In vitro invasion was measured by Matrigel invasion assay. We also analyzed tumorigenicity in heparanase-suppressed HepG2 cells in nude mice. We found that siRNA-1 (1214-1232) and siRNA-3 (611-629) targeting heparanase significantly downregulated the expression of heparanase in HepG2 liver cancer cells. Compared with its controls, siRNA-1 or siRNA-3 vectors efficiently inhibited the proliferation and invasion of HepG2 liver cancer cells in vitro and tumorigenesis in vivo. These results suggest that heparanase-specific RNA interference has potential value as a novel therapeutic agent for human liver cancer.
\end{abstract}

Correspondence to: Dr Shi-Ming Yang or Dr Chuan Li, Institute of Gastroenterology, Third Military Medical University, Chongqing 400038, P.R. China

E-mail: shimingyang@yahoo.com

E-mail: jessica0023@sina.com

Abbreviations: HCC, hepatocellular carcinoma; Hpa, heparanase; ECM, extracellular matrix; BM, basement membrane; siRNA, small interfering RNA; RNAi, RNA interference

Key words: heparanase, RNA interference, invasion, tumorigenesis, liver cancer, small interfering RNA

\section{Introduction}

Hepatocellular carcinoma (HCC) is one of the leading causes of worldwide cancer mortality, with an estimated 1 million deaths annually, and a 5-year survival rate of less than 5\% (1). The incidence has been reported to be 120 per 100,000 in Asia and Africa, compared with 4 to 15 per 100,000 in Western countries (2). Early detection of HCC can be difficult, as most of the patients who develop this tumor have no symptoms. Due to rapid intrahepatic and extrahepatic metastasis, most patients with HCC are not diagnosed until the disease has reached an advanced stage, leading to a poor prognosis for this disorder. In past decades, extensive studies have been performed to investigate the biochemical and molecular mechanisms involved in cancer metastasis and recurrence and to explore effective anti-metastasis therapy. These investigations have resulted in improved prognosis for many types of cancer but have had little effect on HCC patients.

Heparanase (Hpa) is the only endo- $\beta$-D-glucuronidase that can cleave heparan sulfate proteoglycans (HSPGs) and has been implicated in tumor angiogenesis and metastasis (3-6). The basement membrane (BM) and extracellular matrix (ECM) act as hurdles for metastasis by limiting the invasion and spread of tumor cells (7). Heparanase, produced by malignant tumor cells, can mediate the degradation of HSPGs in ECM and BM. Besides its involvement in cell migration from the vasculature, heparanase has also been shown to elicit an angiogenic response by releasing heparin-binding angiogenic factors sequestered in the BM and ECM, such as bFGF, VEGF, and HGF (8). A pronounced correlation between heparanase expression and tumor microvessel density has been reported $(9,10)$. Therefore, heparanase may facilitate tumor cell invasion and neovascularization, which are critical steps in cancer metastasis. Since the heparanase gene was cloned by four independent groups (3-6), a large number of studies have clearly linked heparanase expression to the process of tumorigenesis and invasion in a wide number of cancers, including gastric, liver, colon, pancreatic, esophageal, breast, bladder, prostate, brain, thyroid, ovary, lung, and acute myeloid leukemia (11). The overexpression of the heparanase cDNA in tumor cells with a low metastatic ability 
conferred a high metastatic potential in experimental animals, resulting in an increased rate of mortality (6). These studies show that heparanase holds promise as a potential target for inhibiting tumor metastasis and angiogenesis.

Since its discovery in 1998 (12), RNA interference (RNAi) has rapidly developed into one of the most widely applied technologies in molecular and cellular research. RNAi is a process of sequence-specific post-transcriptional gene silencing. When small interfering RNA (siRNA) is introduced into mammalian cells, sequence specific destruction of endogenous target mRNA occurs, and the expression of the target gene is suppressed. Thus, RNAi has distinct advantages over other methods in inhibiting gene expression, such as high specificity, efficacy, stability, and reproducibility, and is one of the simplest and most effective tools to explore gene function (13-15).

In order to explore the effect of heparanase downregulation on the invasion and tumorigenesis of HepG2 liver cancer cells, using an online siRNA tool, we designed three siRNA sequences to target heparanase and cloned them into the pGenesil-1 vector. The siRNA vectors were then transfected into HepG2 cells. The results demonstrated that two siRNA sequences could significantly inhibit the proliferation, invasion, and tumorigenesis of HepG2 cells in vitro and in vivo through the downregulation of heparanase expression. These results indicate that heparanase silencing may serve as an efficient cancer therapy for human HCC.

\section{Materials and methods}

Cell lines and animals. The human liver cancer cell line HepG2 was purchased from the American Type Culture Collection (Rockville, MD). Mouse embryo fibroblasts, NIH3T3 cells were a generous gift of Dr Guangping Liang (Institute of Burn Research, Southwest Hospital, Third Military Medical University, Chongqing China). The cells were cultured in Dulbecco's modified Eagle's medium (Gibco-BRL, Carlsbad, CA) supplemented with $10 \%$ fetal bovine serum (Gibco-BRL). All cultures were maintained at $37^{\circ} \mathrm{C}$ in a humidified atmosphere containing $5 \% \mathrm{CO}_{2}$. Twelve nude male mice (4 weeks of age) were purchased from the Animal Department, Third Military Medical University, Chongqing, China and were maintained in a specific pathogen-free environment. Animal studies were performed in compliance with the local ethics committee of the Third Military Medical University.

Construction of the siRNA expression vector. The pGenesil-1 vector containing the U6 promoter region was purchased from the Genesil Biotechnology Corporation (Wuhan, China). Using an online siRNA tool (www.genescript.com), we designed three different short interfering RNAs (siRNA) targeting heparanase and one scrambled siRNA (used as a negative control). The three nucleotide sequences of the human heparanase siRNAs were chemically synthesized (Takara, Dalian, China) as follows: 5'-GATCCGTTGAACAGAAGAG ATAGCCTCAAGACGGGCTATCTCTTCTGTTCAATTTT TTA-3' (sense) and 3'-GCAACTTGTCTTCTCTATCGGAGT TCTGCCCGATAGAGAAGACAAGTTAAAAAATTCGA-5' (antisense) for siRNA-1 (target located at 1214-1232 encoding region of human heparanase); 5'-GATCCGTCAATGGTGA CGGACAGGATCAAGACGTCCTGTCCGTCACCATTGAT
TTTTTA-3' (sense) and 3'-GCAGTTACCACTGCCTGTCCT AGTTCTGCAGGACAGGCAGTGGTAACTAAAAAATT CGA-5' (antisense) for siRNA-2 (target located at 167-185 encoding region of human heparanase); 5'-GATCCGTAGTCC AGGAGCAACTGAGTCAAGACGCTCAGTTGCTCCTGG ACTATTTTTT A-3' (sense) and 3'-GCATCAGGTCCTCGTT GACTCAGTTCTGCGAGTCAACGAGGACCTGATAAAA AATTCGA-5' (antisense) for siRNA-3 (target located at 611-629 encoding region of human heparanase). In addition, oligonucleotides 5'-GATCCGACACCTATACAACGGTAGT TCAAGACGACTACCGTTGTATAGGTGTTTTTTTA-3' (sense) and 3'-GCTGTGATATGTTGCCATCAAGTTCTGCT GATGGCAACATATCCACAAAAAAATTCG A-5' (antisense) were designed to serve as controls (siRNA-N). In each pair of oligonucleotides, BamHI and HindIII restriction sites were included at the $5^{\prime}$ and $3^{\prime}$ ends, respectively.

Stable transfection. HepG2 cells were inoculated at a density of $1 \times 10^{5}$ cells/well in 24 -well plates. The cells were grown to $70-80 \%$ confluence and transfected with a recombinant vector containing the siRNA sequence against heparanase in the presence of DOTAP liposome (Roche, Mannheim, Germany) following the manufacturer's instructions. After selection in G418 (300 $\mu \mathrm{g} / \mathrm{ml}$, Gibco-BRL, Gaithersburg, MD) for 4 weeks, surviving colonies were isolated and expanded.

SYBR green-based real-time quantitative RT-PCR. RNA was isolated from $5 \times 10^{5}$ tumor cells with TRIzol (Invitrogen, Carlsbad, CA) according to the manufacturer's instructions and quantified by spectrophotometry. After oligo(dT)-primer reverse transcription of $500 \mathrm{ng}$ of total RNA, the resulting single-stranded cDNA was amplified using the Perfect RealTime RT-PCR kit (Takara). The following primers were used in this study: 5'-GAATGGCCCTACCAGGAGCA-3' (sense) and AACGCATTTAGGCCAAAGATCAAG (antisense) for heparanase; 5'-GTTGCGTTACACCCTTTCTTGACA-3' (sense) and 5'-GCACGAAGGCTCATCATTCAAAA-3' (antisense) for human $\beta$-actin. Real-time quantitative PCR experiments were performed using the SYBR Green PCR Core kit (Applied Biosystems, Foster City, CA, USA) according to the vendor's instructions and an ABI 7900HT (Applied Biosystems) real-time PCR instrument with the following settings: initial denaturation at $95^{\circ} \mathrm{C}$ followed by 40 cycles of denaturation for $5 \mathrm{sec}$ at $95^{\circ} \mathrm{C}$, annealing for $30 \mathrm{sec}$ at $64^{\circ} \mathrm{C}$, and extension for $15 \mathrm{sec}$ at $72^{\circ} \mathrm{C}$.

Western blot analysis. Proteins in the cell extracts were separated by sodium dodecyl sulfate-polyacrylamide gel electrophoresis (SDS-PAGE) using an $8 \%$ polyacrylamide gel and transferred onto a nitrocellulose membrane. The membrane was incubated with $5 \%$ non-fat milk in PBS, followed by anti-heparanase $\mathrm{mAb}$ (Santa Cruz Biotechnology, CA, USA) or anti- $\beta$-actin mAb (Sigma, St. Louis, MO) for $2 \mathrm{~h}$ at RT. The membranes were washed and incubated with an alkaline phosphatase-conjugated goat anti-mouse IgG antibody (Amersham Biosciences, Buckinghamshire, England) for $1 \mathrm{~h}$ at RT. Immunoreactive bands were detected using the ECL Western blot analysis system (Amersham Biosciences).

Proliferation assay. MTT experiments were carried to detect the proliferative capacity of HepG2 cells that were transfected with 


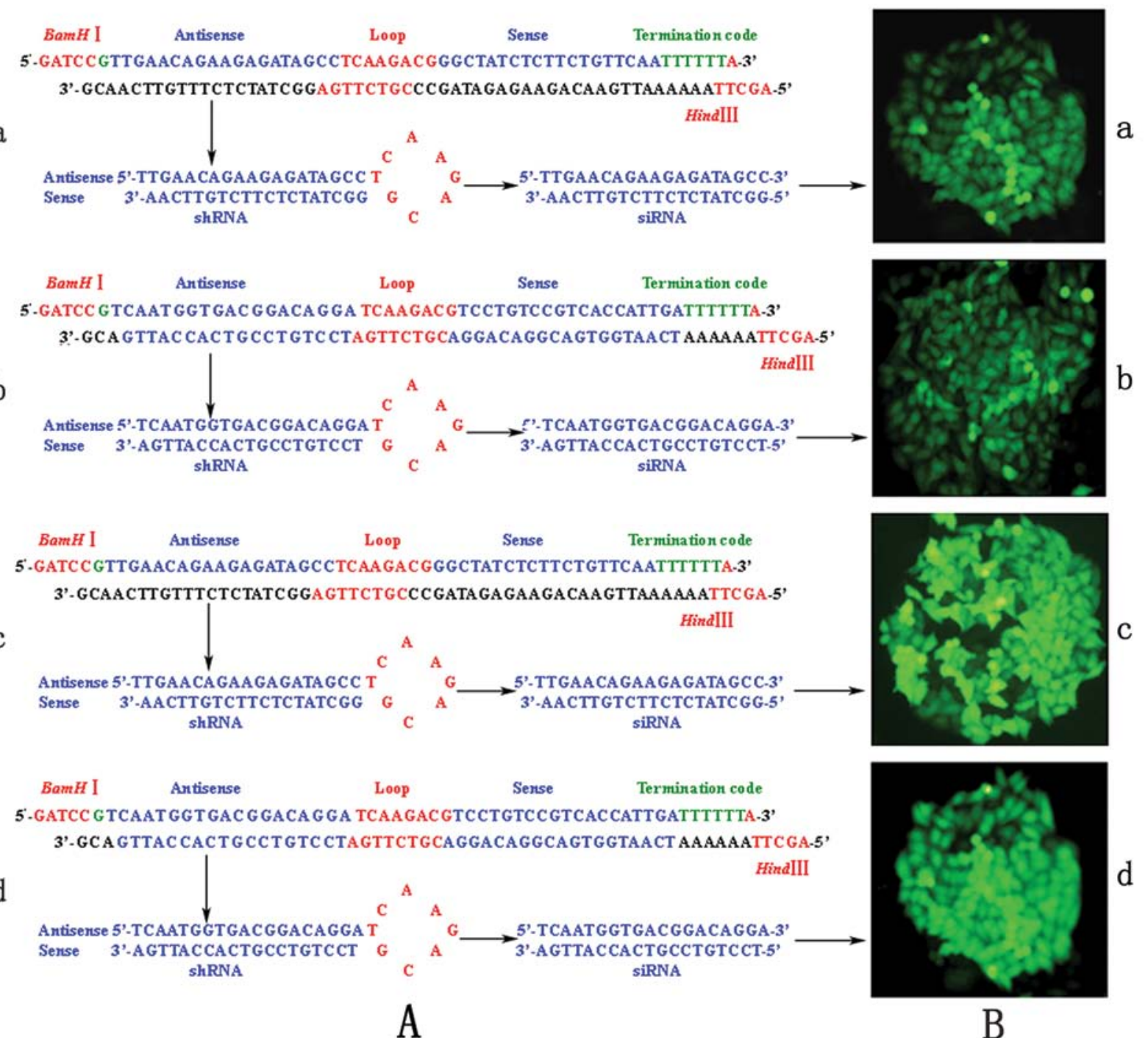

Figure 1. RNA interfering sequences targeting different encoding regions of human heparanase and clone formation after selection by G418. (A) Design of RNA interfering sequences targeting different encoding regions of human heparanase. Target located at 1214-1232 encoding region of human heparanase (siRNA-1) (A-a); 167-185 (siRNA-2) (A-b) and 611-629 (siRNA-3) (A-c). Unrelated sequence was designed as control (siRNA-N) (A-d). (B) Enhanced green fluorescence protein (EGFP) detected in clone formation of HepG2 transfeced with different vectors after selected by G418. HepG2 liver cancer cells were transfected with siRNA-1 (HepG2/RNAi-1) (B-a), siRNA-2 (HepG2/RNAi-2) (B-b), siRNA-3 (HepG2/RNAi-3) (B-c) or siRNA-N (HepG2/RNAi-N) (B-d), respectively and EGFP was detected by fluorescence microscopy in each clone.

siRNA of heparanase or control vectors according to our prior study (16). In brief, 1000 HepG2 cells transfected with control or siRNA of heparanase were plated onto 96 -well culture dishes. At each time point, $20 \mu \mathrm{l}(5 \mathrm{mg} / \mathrm{ml}) 3$-(4,5-dimethylthiazoyl-2)-2,5diphenyltetrazolium bromide (MTT) was added into each well and incubated at $37^{\circ} \mathrm{C}$ for $4 \mathrm{~h}$. The media were carefully aspirated, and $200 \mu \mathrm{l}$ dimethyl sulfoxide (DMSO) was added to each well and pipetted up and down to dissolve crystals. The intensity of dissolved formazan crystal was measured at $490 \mathrm{~nm}$. Each experiment was repeated three times.

Cell cycle analysis by flow cytometry. Cell cycle distribution was detected by flow cytometry as previously reported (16). Briefly, cells at $50 \%$ confluence or less were fixed in $70 \%$ icecold ethanol for at least $24 \mathrm{~h}$. The cells were washed with PBS, treated with RNase A $(75 \mathrm{U} / \mathrm{ml})$ for $30 \mathrm{~min}$ at $37^{\circ} \mathrm{C}$, washed again in PBS, and resuspended in PBS containing $15 \mathrm{mg} / \mathrm{ml}$ propidium iodide. A minimum of $2 \times 10^{4}$ cells were analyzed with a Coulter Epics Profile II (Coulter Electronics, Inc., Hialeah, FL, USA). The proliferative index (PI) was calculated according to the following formula: $\left[\left(S+G_{2} M\right) /\left(G_{0} / G_{1}+S+G_{2} M\right)\right]$ $\mathrm{x} 100 \%$.

Clone formation test. In order to test the clone-formation capacity of HepG2 cells transfected with siRNA of heparanase or control vectors, cells were seeded in $35 \mathrm{~mm}$ plates $\left(1.5 \times 10^{2}\right.$ cells/plate). Colonies were allowed to grow for 14 days. The medium was discarded, and each well was washed twice with phosphate buffered saline (PBS). The cells were fixed in methanol for $15 \mathrm{~min}$ and then stained with Giemsa. Clones containing at least 50 cells were counted using an inverse microscope.

Matrigel invasion assay. Tumor cells $\left(3 \times 10^{5}\right.$ cells in $1 \mathrm{ml}$ DMEM containing $0.1 \%$ BSA) were assayed in triplicate for 
Matrigel invasion at $37^{\circ} \mathrm{C}$ in a humidified incubator $(95 \%$ air, 5\% $\mathrm{CO}_{2}$ ) for $30 \mathrm{~min}$, using Transwell chambers ( $\mathrm{R} \& \mathrm{D}$ Systems China Co. Ltd., Shanghai, China) and polycarbonate filters $(13 \mathrm{~mm}$ in diameter, $8 \mu \mathrm{m}$ pore size; Costar Scientific, Cambridge, MA) coated with Matrigel. Medium conditioned by NIH3T3 fibroblasts was used as a chemoattractant and placed in the lower compartment of the Transwell chamber. As a negative control, serum-free DMEM containing 0.1\% BSA was placed in the lower compartment of the Transwell chamber instead of the chemoattractant. After $24 \mathrm{~h}$, the filters were removed, and the cells on the lower surface of the filter were stained with Giemsa. The cells that migrated through the membrane to the lower surface of the filter were counted in five microscopic fields, selected at random (x200 magnification), for each well (17).

Tumorigenicity in nude mice. Twelve nude male mice, 4 weeks old, were purchased from the Animal Department, Third Military Medical University, China, and were randomly divided into four groups (3 mice per group): HepG2/RNAi-1, HepG2/ RNAi-3, HepG2/RNAi-N, and HepG2 parental cells. Animals were maintained under the guidance set forth in the 'Guide for the Care and Use of Laboratory Animals' by the National Laboratory Animals Administration Council, China. For subcutaneous cancer cell inoculation, HepG2 cells in exponential growth phase were collected, and single cell suspensions at a density of $5 \times 10^{7}$ cells $/ \mathrm{ml}$ were prepared. Single cell suspensions (200 $\mu$ l) were subcutaneously inoculated into rear portions of the right inguinal grooves of nude mice. After one month, the mean tumor volumes were measured and calculated according to the formula $v=a^{2} b^{2} / 2$ ('a' represents the maximum diameter of the tumor, and ' $b$ ' represents the vertical diameter of the maximum diameter).

Statistical analysis. Mean \pm standard deviation (SD) was calculated from the results of at least three replicates within one experiment or from at least three comparable experiments. The student's t-test was used to determine the significance $(p<0.05)$ of the differences between the mean values. All statistical analyses were carried out with the SPSS 11.5 software.

\section{Results}

Construction and transduction of siRNA against heparanase. Small interfering RNA (siRNA) can silence gene expression in a sequence-specific manner. Because different siRNAs targeting the same gene vary in efficiency, we designed three siRNAs against the open reading frame of human heparanase mRNA, as illustrated in Fig. 1. Each pair of oligonucleotides $(100 \mathrm{pmol})$ was annealed by incubation at $95^{\circ} \mathrm{C}$ for $5 \mathrm{~min}$ and cooled slowly. This mixture $(1 \mu \mathrm{l})$ was then inserted between the BamHI and HindIII sites of the pGenesil-1 vector. The resulting siRNA-containing plasmids were named pRNAi-1, pRNAi-2, pRNAi-3 and pRNAi-N. The plasmids were sequenced in order to verify that the siRNA sequence was successfully cloned in the recombinant vectors (data not shown).

The siRNA expression vectors were then transfected into HepG2 liver cancer cells in the presence of DOTAP liposome (Roche, Mannheim, Germany). After selection in G418 (300 $\mu \mathrm{g} / \mathrm{ml}$, Gibco-BRL, Gaithersburg, MD) for 4

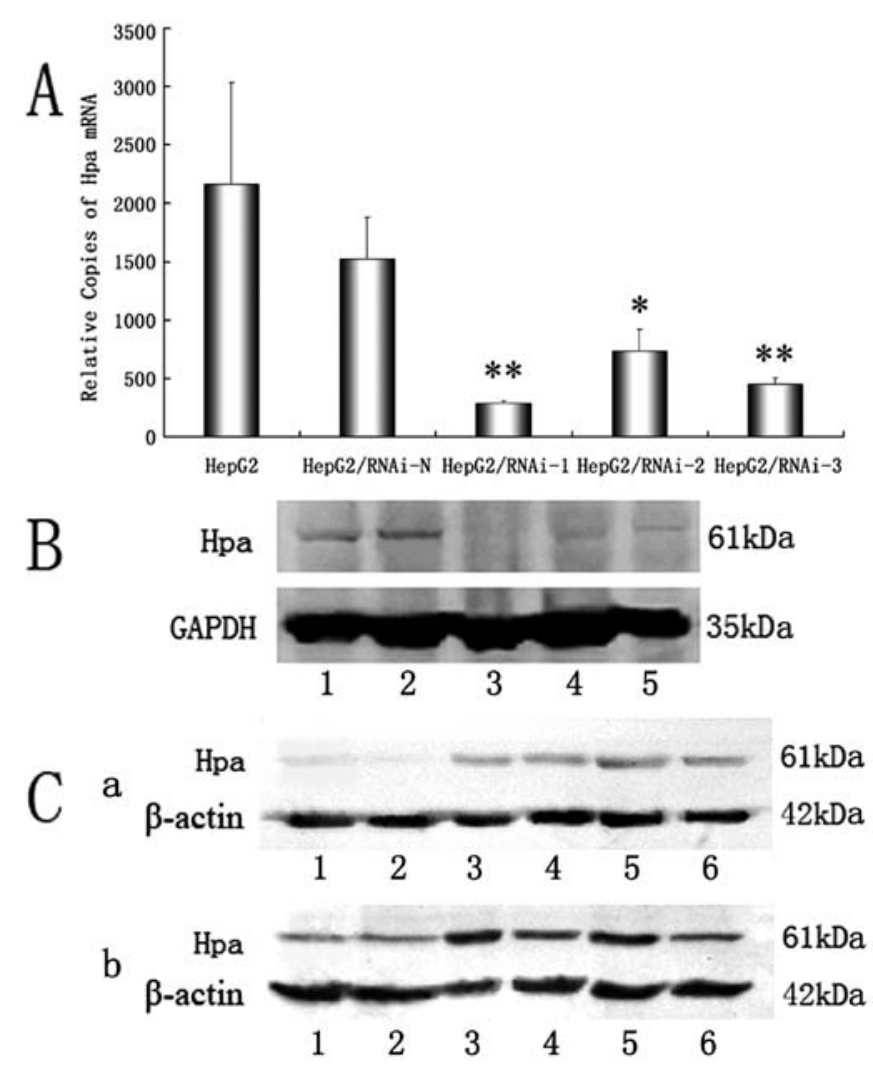

Figure 2. Silencing heparanase downregulated the expression of heparanase in HepG2 liver cancer cells. (A) Expression of heparanase mRNA detected by real-time RT-PCR. Compared with heparanase mRNA in HepG2 and HepG2/ RNAi-N cells, heparanase mRNA in HepG2/RNAi-1, HepG2/RNAi-2 and HepG2/RNAi-3 cells was significantly downregulated ( $\left.\mathrm{p}<0.05,{ }^{* *} \mathrm{p}<0.01\right)$. (B) Expression of heparanase protein detected by Western blotting. 1, HepG2; 2, HepG2/RNAi-N; 3, HepG2/RNAi-1; 4, HepG2/RNAi-2; 5, HepG2/RNAi-3. (C) Expression of heparanase protein in different generations of HepG2/ RNAi-1 and HepG2/RNAi-3 cells. (C-a) Expression of heparanase protein in different generations of HepG2/RNAi-1. 1, The 5th generation HepG2/ RNAi-1; 2, The 10th generation HepG2/RNAi-1; 3, The 15th generation HepG2/RNAi-1; 4, The 19th generation HepG2/RNAi-1; 5, HepG2/RNAi-N; 6: HepG2. (C-b) Expression of heparanase protein in different generations of HepG2/RNAi-3. 1, The 5th generation HepG2/RNAi-3; 2, The 10th generation HepG2/RNAi-3; 3, The 15th generation HepG2/RNAi-3; 4, The 19th generation HepG2/RNAi-3; 5, HepG2/RNAi-N; 6: HepG2.

weeks, drug resistant clones were isolated and named HepG2/ RNAi-N, HepG2/RNAi-1, HepG2/RNAi-2, HepG2/RNAi-3, respectively. pGenesil-1 encodes enhanced green fluorescence protein (EGFP), which we visualized by fluorescence microscopy, to monitor successful transduction of the heparanase siRNA expressing vectors into the HepG2 cells. As shown in Fig. 1, EGFP was detected in cells transfected with recombinant DNA, which indicated that the transductions were successful.

The effect of heparanase siRNA on the expression of heparanase in Hep 22 cells. SYBR green-based real-time quantitative RT-PCR was used to analyze the amounts of heparanase mRNA isolated from HepG2, HepG2/RNAi-N, HepG2/RNAi-1, HepG2/RNAi-2, and HepG2/RNAi-3 cells. Relative heparanase mRNA was calculated and shown in Fig. 2A. These results 

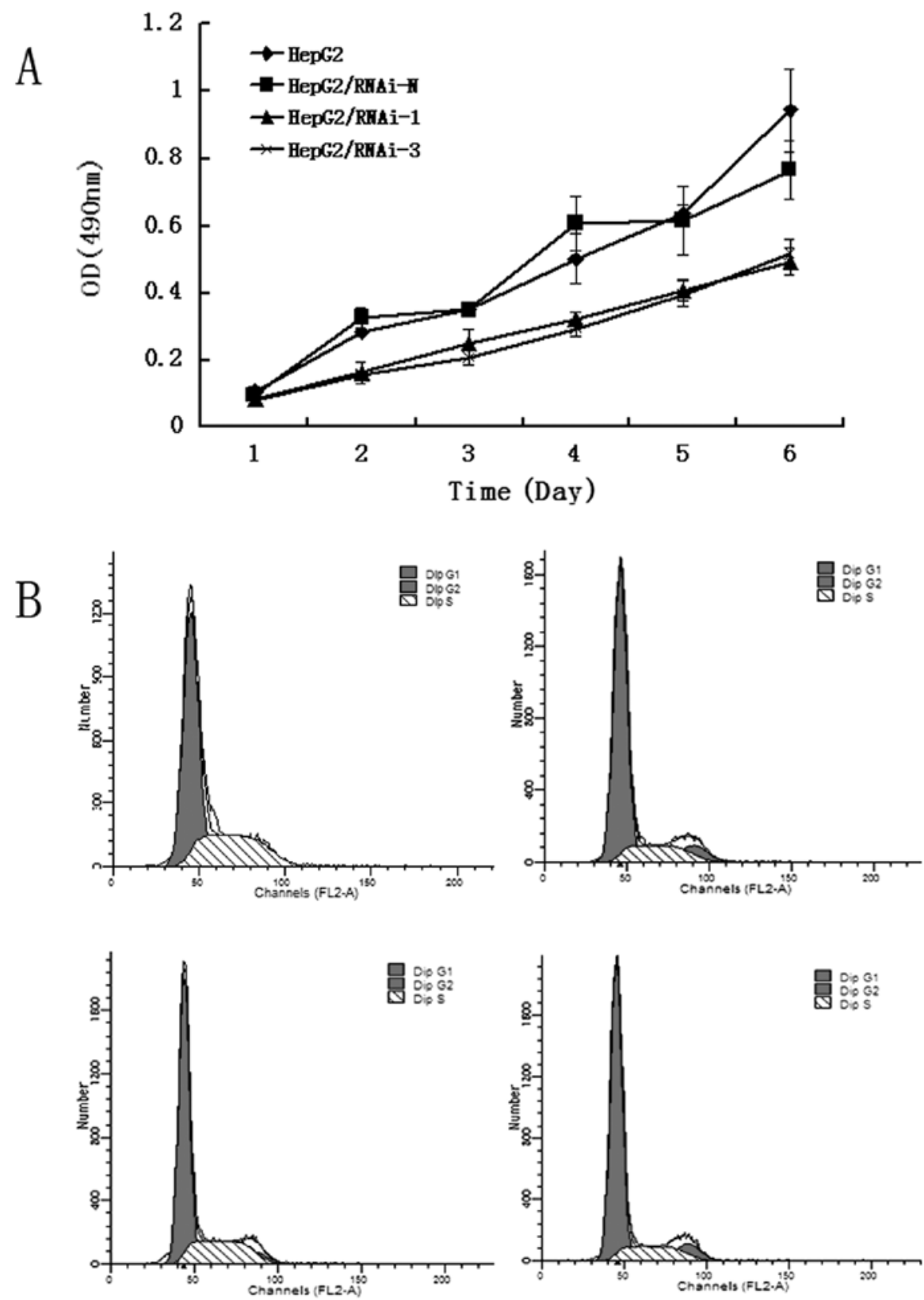

Figure 3. RNA interference of heparanase inhibited the growth and changed cell cycle distribution of HepG2. (A) RNAi of heparnase inhibited the growth of HepG2 transfected with RNAi-1 and RNAi-3. (B) RNAi of heparanase arrested HepG2 cells in the $\mathrm{G}_{0} / \mathrm{G}_{1}$ phase.

suggest that siRNA-1, siRNA-3, and less so, siRNA-2 can significantly attenuate heparanase activity in HepG2 cells at the mRNA level.

To determine the effect of these three RNAi sequences on the protein expression of heparanase, HepG2, HepG2/RNAi-N, HepG2/RNAi-1,HepG2/RNAi-2 and HepG2/RNAi-3 cells were examined by Western blotting. The protein levels of heparanase were standardized to GAPDH levels. siRNA-N had almost no effect on the protein expression of heparanase, whereas siRNA-1 and siRNA-3 appeared to decrease the protein expression of heparanase by $57 \%$ and $71 \%$, respectively (Fig. 2B). Therefore, we chose HepG2/RNAi-1 and HepG2/RNAi-3 cells to conduct our following experiments.

To determine how long the effect of RNAi would last in liver cancer cells, we performed a Western blot analysis to determine heparanase expression in various generations (5th,
Table I. Effect of heparanase RNAi on distribution of cell cycle of HepG2 cells.

\begin{tabular}{lcccc}
\hline Cell lines & $\mathrm{G}_{0} / \mathrm{G}_{1}$ & $\mathrm{~S}$ & $\mathrm{G}_{2} / \mathrm{M}$ & $\mathrm{PI}$ \\
\hline HepG2 & 65.73 & 33.86 & 0.41 & 34.27 \\
HepG2/RNAi-N & 67.33 & 27.51 & 6.16 & 33.67 \\
HepG2/RNAi-1 & 73.93 & 17.72 & 8.35 & 26.07 \\
HepG2/RNAi-3 & 74.72 & 16.74 & 8.64 & 25.38 \\
\hline
\end{tabular}

10th, 15th and 19th) of HepG2/RNAi-1 and HepG2/RNAi-3 cells. The result showed that the siRNA was stable for the first 10 generations after transfection. After the 10th generation, the 


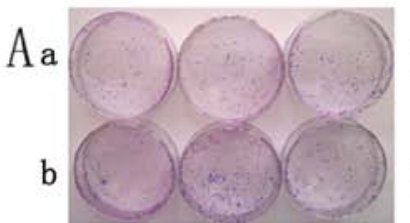

C

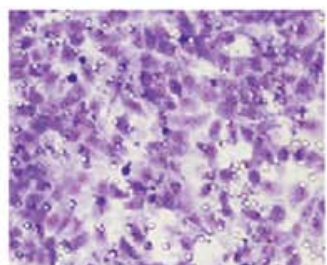

HepG2

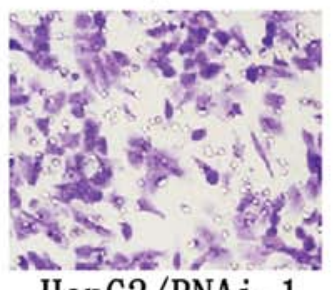

HepG2/RNAi-1
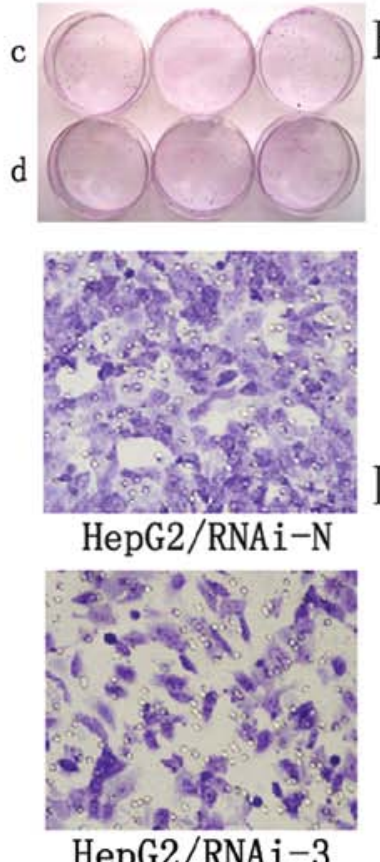

HepG2/RNAi-3
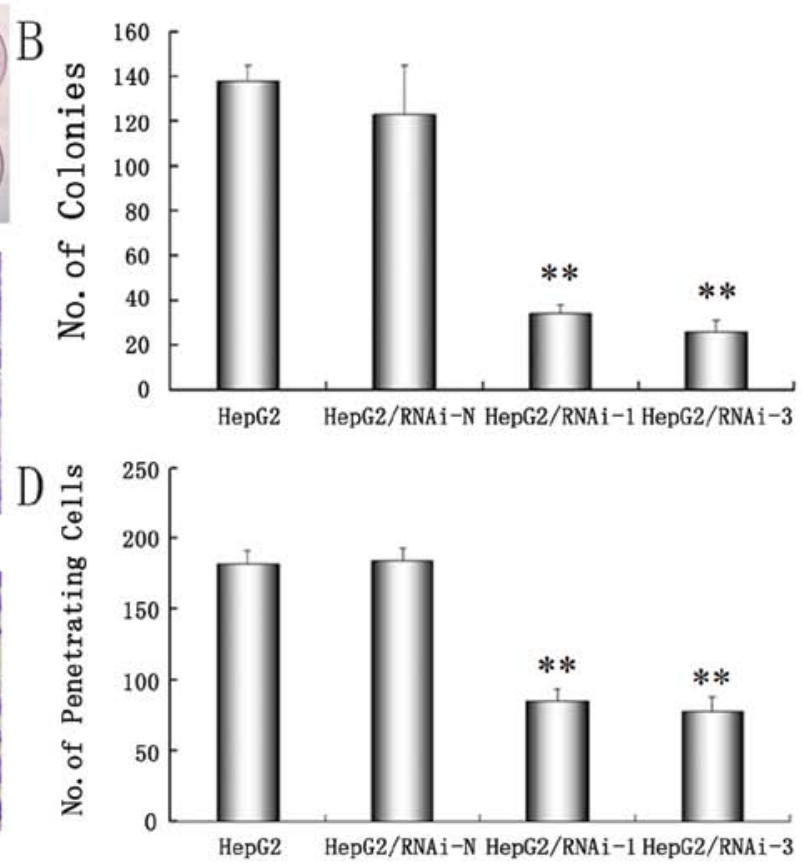

Figure 4. RNA interference of heparanase attenuated clone formation and invasion of HepG2 liver cancer cells in vitro. (A and B) The ability of a single cell to form a clone detected by plate clone formation. (A-a) HepG2; (A-b) HepG2/RNAi-N; (A-c) HepG2/RNAi-1; (A-d) HepG2/RNAi-3. Compared with HepG2 and HepG2/RNAi-N cells, ${ }^{* *}$ p $<0.01$. (C and D) In vitro invasion of HepG2 detected by transwell analysis. Compared with HepG2 and HepG2/RNAi-N cells, ${ }^{* *}$ p $<0.01$.
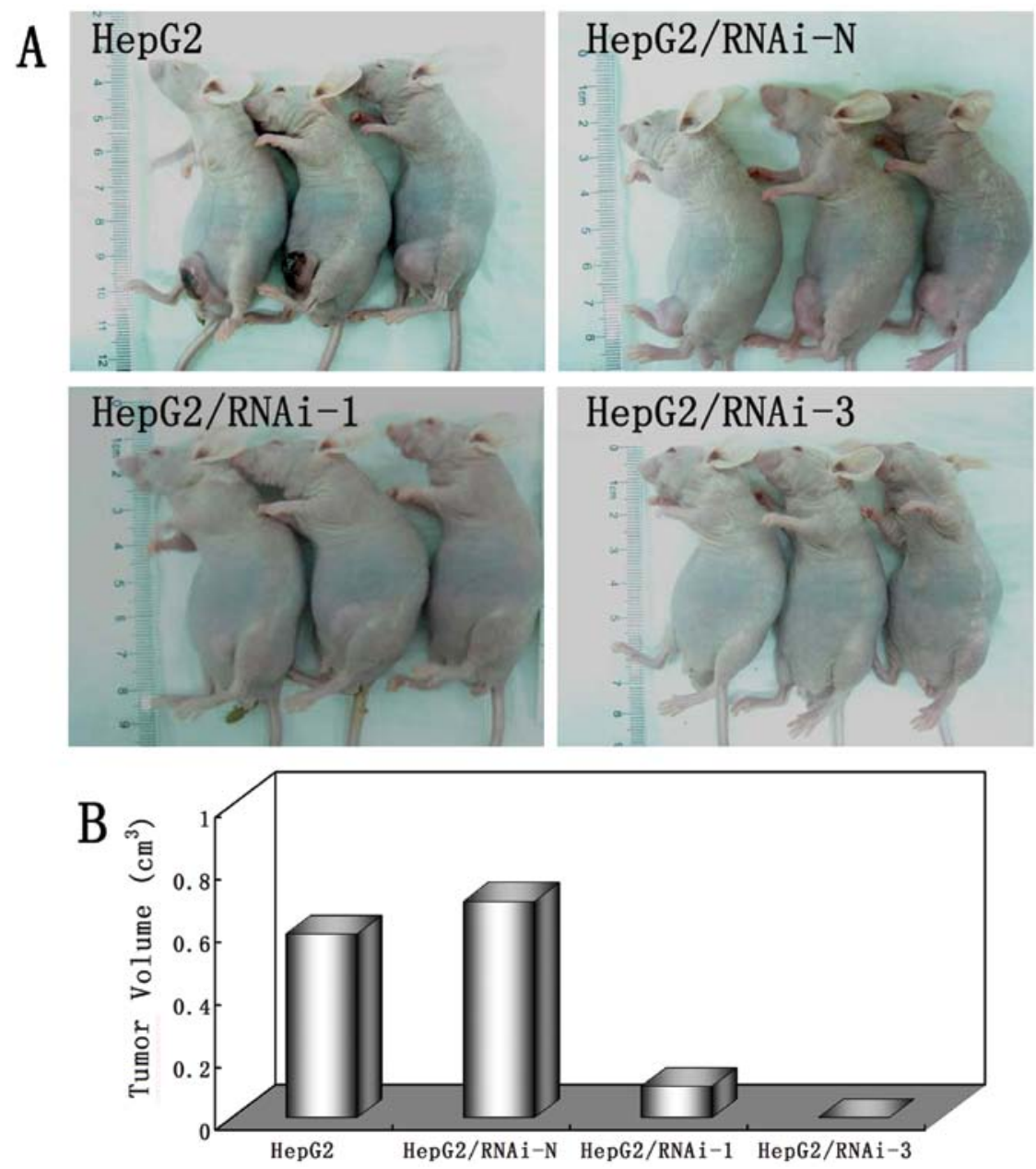

Figure 5. RNA interference of heparanase inhibited tumorigenicity of HepG2 in nude mice. (A) The ability of tumorigenicity of different HepG2 cells in nude mice. (B) Tumor volume of different HepG2 cells in nude mice after 30 days. 
effect of RNAi was rapidly lost (Fig. 2C). Therefore, we chose cells that were close to the 5 th generation to conduct our other experiments.

Heparanase siRNA significantly inhibits proliferation. We tested the effect of heparanase RNAi on the proliferation of HepG2 cells by the MTT assay. As illustrated in Fig. 3A, HepG2/RNAi-1 had a similar growth rate as HepG2/RNAi-3 cells, and both growth rates were obviously slower than parental HepG2 cells and HepG2/RNAi-N cells. These results suggest that heparanase RNAi suppresses heparanase-associated cell proliferation in vitro.

The heparanase siRNA were also found to have an effect on the cell cycle (Fig. 3B and Table I). Flow cytometry showed that an increased percentage of cells were in the $G_{0} / G_{1}$ phase and a decreased percentage of cells in the proliferative index (PI) in HepG2/RNAi-1 and HepG2/RNAi-3 cells, compared with their parental cells and HepG2/RNAi-N cells (Table I). This result indicates that heparanase siRNA can arrest cells in the $\mathrm{G}_{0} / \mathrm{G}_{1}$ phase.

Effect of heparanase siRNA on clone formation of HepG2 cells. We also adopted the clone formation test to determine the change in proliferation ability of a single HepG2 cell before and after heparanase RNAi. The result demonstrated that the single cell clone formation capacity of HepG2/RNAi-1 and HepG2/ RNAi-3 cells was obviously lower than that of parental HepG2 and HepG2/RNAi-N cells $(34 \pm 4,26 \pm 5$ vs. $138 \pm 7,123 \pm 22$, $\mathrm{p}<0.01$, Fig. $4 \mathrm{~A}$ and B). This indicates that downregulation of heparanase can decrease the cloning efficiency of HepG2 cells.

Heparanase siRNA significantly inhibits the invasive ability of tumor cells in vitro. Because heparanase plays a role in cell invasion through the degradation of HSPG in the ECM and BM $(18,19)$, we tested the effect of heparanase RNAi on the invasive ability of HepG2 cells using the Matrigel invasion assay. As shown in Fig. 4C and D, the ability of HepG2/ RNAi-1 or HepG2/RNAi-3 to invade through Matrigel-coated filters was significantly inhibited compared with the parental HepG2 cells and HepG2/RNAi-N $(85 \pm 9,78 \pm 10$ vs. $182 \pm 10$, $184 \pm 9, \mathrm{p}<0.01)$.

Heparanase siRNA significantly inhibits tumorigenesis in vivo. We have demonstrated that heparanase RNAi can inhibit the proliferation of HepG2 cells in vitro. Whether heparanase siRNA can decrease the tumorigenesis of HepG2 cells in vivo remained unknown. A xenograft model was employed to compare the tumorigenicity of HepG2 cells before and after heparanase inhibition. Subcutaneous tumor nodes of different groups became palpable almost simultaneously, 7 days after transplantation, with the exception of the HepG2/RNAi-3 group. Finally, tumor graft volume was measured after 30 days. The results showed that HepG2 and HepG2/RNAi-N cells had a tumor formation ratio of $100 \%$. Although HepG2/RNAi-1 cells also had a tumor formation ratio of $100 \%$, its subcutaneous tumor volume was much smaller than of parental HepG2 and HepG2/RNAi-N cells $(0.099 \pm 0.030$ vs. $0.585 \pm 0.135,0.690 \pm 0.099, \mathrm{p}<0.01)$. No subcutaneous tumors formed in the HepG2/RNAi-3 cells (Fig. 5A and B).

\section{Discussion}

Numerous studies have demonstrated that heparanase is upregulated in a large number of malignant tumors, including liver cancer. Overexpression of heparanase directly correlated with an increase in cellular invasion, angiogenesis, and metastasis $(6,20)$. Recently, Chen and colleagues detected heparanase expression in liver cancer by tissue microarray (21). They found that heparanase was significantly higher in HCC tissues than in adjacent tissues. Heparanase expression correlated with clinical stage, portal vein tumor emboli, multiple tumor nodes, and tumor diameter. These results indicated that overexpression of heparanase may play an important role in hepatocarcinogenesis, progression, and metastases of HCC and supported the notion that heparanase could serve as a potential target for HCC therapy.

In the past decade, potent heparanase inhibitors, such as small molecule, sugar, natural product inhibitors, and neutralizing antibodies, have shown promising efficacy at the pre-clinical stage. One representative of these inhibitors, PI-88, is being evaluated in a multicenter phase II or III clinical trial $(22,23)$. However, due to the multiple biologic activities of these inhibitors, the mechanism of their anti-tumor activity and their relation to heparanase inhibition are not clear (24). Therefore, the application of these inhibitors in the clinic may require further study.

RNA interference is a post-transcriptional mechanism of gene silencing through chromatin remodeling, inhibition of protein translation, or direct mRNA degradation. Genetic silencing of heparanase expression by RNAi may be a highly specific, promising alternative (25). Recent studies have attempted to use a siRNA approach to inhibit heparanase. Edovitsky et al demonstrated that siRNA-mediated gene silencing resulted in the downregulation of heparanase enzymatic activity, tumor invasiveness, angiogenesis, and metastasis (24). Zhang and colleagues reported that silencing heparanase in breast cancer cells could decrease their invasion and adhesion in vitro. In an MDA-MB-435 cell xenograft model, tumors treated with siRNA were less vascularized and less metastatic than the control group (26). These results indicated that the reduction of heparanase expression led to the inhibition of invasion, metastasis, and angiogenesis of tumor cells, both in vitro and in vivo.

In the present study, we designed three different siRNA vectors targeting three different sequences of the heparanase RNA. The results demonstrated that RNAi-1 (1214-1232: 5'-GGCTATCTCTTCTGTTCAA-3') and RNAi-3 (611-629: 5'-CTCAGTTGCTCCTGGACTA-3') dramatically knocked down the expression of heparanase mRNA and protein in a sequence-specific manner when compared with the negative control.

The relationship between heparanase and cell proliferation has, thus far, been unclear. Edovitsky et al found that mock- and siRNA-transfected B16-BL6 cells had the same rate of proliferation in vitro (24), whereas Zhang et al demonstrated that the proliferation of MDA-MB-435 cells was significantly inhibited in vitro when heparanase was targeted by siRNA (26). In the present study, we found that the proliferative capacity of HepG2 cells was significantly decreased after downregulation of heparanase. This result was further confirmed by cell cycle analysis. 
The flow cytometry result showed an increase in the percentage of cells in $\mathrm{G}_{0} / \mathrm{G}_{1}$ phase and a decrease in the percentage of cells in the proliferative index (PI) in siRNA-1 and siRNA-3 transfected HepG2 cells. A previous study showed that RNAimediated heparin inhibition led to expression changes of many genes related to the cell cycle and proliferation (26). Zhang and colleagues studied the effect of heparanase siRNA on the gene expression signature of tumor cells. They found that proteoglycan 2 (PRG2), which can reduce cell proliferation by inhibiting the response of cells to growth factors (27), was the most upregulated gene. Meanwhile, they also found that cell division cycle 16 (CDC16), which is an important component of the anaphase promoting complex (28), was the most downregulated gene. These results support the idea that siRNA against heparanase could regulate the expression of cell growth and cell cycle related genes, and thus, affect cell proliferation.

Because siRNA efficacy and mode of delivery vary considerably (29), efficient delivery systems should be considered when RNAi is used as a therapeutic tool. Although present studies have supported the great potential of the RNAi technique, a critical step involves obtaining a stable vector with sustained expression of the siRNA. In the present study, the siRNA vector was constructed based on a eukaryotic expression vector and can be maintained in cells for ten generations of stable expression. Its inhibitory effect gradually disappears as cells continue to expand beyond generation 10. Lentivirus, which can infect both dividing and non-dividing cells and allow for long-term gene expression, can extend the range of cells and tumor types in which the heparanase-silencing approach may be practical (30).

Although the effectiveness of RNAi is most likely determined by the accessibility of its target sequences in the intended cells, at present there are no definitively reliable ways to predict or identify the ideal target sequences. In addition, targeting sequences at different locations may result in different efficiencies of knockdown (31). Although we did not test and compare the efficiency of each potential target, we believe that more efficient targets may exist, apart from those listed in our study.

Collectively, the results presented here indicate that RNAi of the heparanase gene can efficiently inhibit the proliferation, invasion (in vitro), and tumorigenicity (in vivo) of HepG2 liver cancer cells by downregulating heparanase expression. These findings demonstrate that heparanase plays a critical role in liver cancer cell proliferation, migration, and invasion and suggest that heparanase gene silencing can be utilized as a novel anticancer method for human liver cancers in the future.

\section{Acknowledgements}

We thank Dr Su YongYue (Institute of Burn Research, Southwest Hospital, Third Military Medical University, Chongqing 400038, P.R. China) for generously providing the plasmid pGenesil-1. This study was supported by grants from the Chongqing Science Fund for Distinguished Young Scholars (CSTC, 2009BA5045).

\section{References}

1. El-Serag HB and Mason AC: Rising incidence of hepatocellular carcinoma in the United States. N Engl J Med 340: 745-750, 1999.
2. Kubicka S, Rudolph KL, Hanke M, Tietze MK, Tillmann HL, Trautwein CE and Manns M: Hepatocellular carcinoma in Germany: a retrospective epidemiological study from a low-endemic area. Liver 20: 312-318, 2000.

3. Hulett MD, Freeman C, Hamdorf BJ, Baker RT, Harris MJ and Parish CR: Cloning of mammalian heparanase, an important enzyme in tumor invasion and metastasis. Nat Med 5: 803-809, 1999.

4. Parish CR, Freeman C, Brown KJ, Francis DJ and Cowden WB: Identification of sulfated oligosaccharide-based inhibitors of tumor growth and metastasis using novel in vitro assays for angiogenesis and heparanase activity. Cancer Res 59: 3433-3441, 1999.

5. Toyoshima $\mathrm{M}$ and Nakajima M: Human heparanase: purification, characterization, cloning and expression. J Biol Chem 274: 24153-24160, 1999.

6. Vlodavsky I, Friedmann Y, Elkin M, Aingorn H, Atzmon R, IshaiMichaeli R, Bitan M, Pappo O, Peretz T, Michal I, Spector L and Pecker I: Mammalian heparanase: gene cloning, expression and function in tumor progression and metastasis. Nat Med 5: 793-802, 1999.

7. Poste G and Fidler I: The pathogensis of cancer metastasis. Nature 283: 139-146, 1980.

8. Elkin M, Ilan N, Ishai-Michaeli R, Friedmann Y, Papo O, Pecker I and Vlodavsky I: Heparanase as mediator of angiogenesis: Mode of action. FASEB J 15: 1661-1663, 2001.

9. Kelly T, Miao HQ, Yang Y, Navarro E, Kussie P, Huang Y, MacLeod V, Casciano J, Joseph L, Zhan FH, Zangari M, Barlogie B, Shaughnessy J and Sanderson RA: High heparanase activity in multiple myeloma is associated with elevated microvessel density. Cancer Res 63: 8749-8756, 2003.

10. Cai YG, Fang DC, Yang SM, Luo YH, Yang MH and Wang DX: Increased expression of heparanase mRNA is correlated with metastasis of gastric carcinoma and is probably correlated with c-met protein. Zhonghua Yi Xue Za Zhi 84: 974-978, 2004.

11. McKenzie EA: Heparanase:a target for drug discovery in cancer and inflammation. Br J Pharmacol 151: 1-14, 2007.

12. Fire A, Xu S, Montgomery MK, Kostas SA, Driver SE and Mello CC: Potent and specific genetic interference by doublestranded RNA in Caenorhabditis elegans. Nature 391: 806-811, 1998.

13. Fraser AG, Kamath RS, Zipperlen P, Martinez-Campos M, Sohrmann $M$ and Ahringer J: Functional genomic analysis of C.elegans chromosome I by systematic RNA interference. Nature 408: 325-330, 2000.

14. Gonczy P,Echeverri C, Oegema K, Coulson A, Jones SJ, Copley RR, Duperon J, Oegema J, Brehm M, Cassin E, Hannak E, Kirkham M, Pichler S, Flohrs K, Goessen A, Leidel S, Alleaume AM, Martin C, Ozlu N, Bork P and Hyman AA: Functional genomic analysis of cell division in C. elegans using RNAi of genes on chromosome III. Nature 408: 331-336, 2000.

15. Kamath RS, Fraser AG, Dong Y, Poulin G, Durbin R, Gotta M, Kanapin A, Le Bot N, Moreno S, Sohrmann M, Welchman DP, Zipperlen P and Ahringer J: Systematic functional analysis of the Caenorhabditis elegans genome using RNAi. Nature 421: 231-237, 2003.

16. Yang SM, Fang DC, Yang JL, Chen L, Luo YH and Liang GP: Antisense human telomerase reverse transcriptase could partially reverse malignant phenotypes of gastric carcinoma cell line in vitro. Eur J Cancer Prev 17: 209-217, 2008.

17. Yu ST, Chen L, Wang HJ, Tang XD, Fang DC and Yang SM: hTERT promotes the invasion of telomerase-negative tumor cells in vitro. Int J Oncol 35: 329-336, 2009.

18. Zhang L, Sullivan P, Suyama J and Marchetti D: Epidermal growth factor-induced heparanase nucleolar localization augments DNA topoisomerase I activity in brain metastatic breast cancer. Mol Cancer Res 8: 278-290, 2010.

19. Tang XD, Wan Y, Chen L, Chen T, Yu ST, Xiong Z, Fang DC, Liang GP and Yang SM: H-2Kb-restricted CTL epitopes from mouse heparanase elicit an antitumor immune response in vivo. Cancer Res 68: 1529-1537, 2008.

20. Cohen I, Pappo O, Elkin M, San T, Bar-Shavit R, Hazan R, Peretz T, Vlodavsky I and Abramovitch R: Heparanase promotes growth, angiogenesis and survival of primary breast tumors. Int J Cancer 118: 1609-1617, 2006.

21. Chen G, Dang YW, Luo DZ, Feng ZB and Tang XL: Expression of heparanase in hepatocellular carcinoma has prognostic significance: a tissue microarray study. Oncol Res 17: 183-189, 2008.

22. Ferro V, Dredge K, Liu LG, Hammond E, Bytheway L, Li CP, Johnstone K, Karoli T, Davis K, Copeman E, Biotech B and Gautam A: PI-88 and novel heparan sulfate mimetics inhibit angiogenesis. Semin Thromb Hemost 33: 557-562, 2007. 
23. Liu CJ, Lee PH, Lin DY, Wu CC, Jeng LB, Lin PW, Mok KT, Lee WC, Yeh HZ, Ho MC, Yang SS, Lee CC, Yu MC, Hu RH, Peng CY, Lai KL, Chang SSC and Chen PJ: Heparanase inhibitor PI-88 as adjuvant therapy for hepatocellular carcinoma after curative resection: A randomized phase II trial for safety and optimal dosage. J Hepatol 50: 958-968, 2009.

24. Edovitsky E, Elkin M, Zcharia E, Peretz and Vlodavsky I: Heparanase gene silencing, tumor invasiveness, angiogenesis, and metastasis. J Natl Cancer Inst 96: 1219-1230, 2004.

25. Pederson T: RNA interference and mRNA silencing, 2004: how far will they reach. Mol Biol Cell 15: 407-410, 2004.

26. Zhang ZH, Chen Y, Zhao HJ, Xie CY, Ding J and Hou YT: Silencing of heparanase by siRNA inhibits tumor metastasis and angiogenesis of human breast cancer in vitro and in vivo. Cancer Biol Ther 6: 587-595, 2007.

27. Laursen LS, Overgaard MT, Weyer K, Boldt HB, Ebbsesn P, Christiansen M, Sorttrup-Jensen L, Giudice LC and Oxvig C: Cell surface targeting of pregnancy-associated plasma protein A proteolytic activity. Reversible adhesion is mediated by two neighboring short consensus repeats. J Biol Chem 277 : 47225-47234, 2002.
28. Yasui K, Arii S, Zhao C, Imoto I, Ueda M, Nagai H, Emi M and Inazawa J: TFDP1, CUL4A, and CDC16 identified as targets for amplification at $13 \mathrm{q} 34$ in hepatocellular carcinomas. Hepatology 35: $1476-1484,2002$

29. Duxbury MS and Whang EE: RNA interference: a practical approach. J Surg Res 117: 3339-3344, 2004.

30. Rubinson DA, Dillon CP, Kwiatkowski AV, Sievers C, Yang L, Kopinja J, Zhang MD, McManus MT, Gertler FB, Scott ML and Van Parijs L: A lentivirus-based system to functionally silence genes in primary mammalian cells, stem cells and transgenic mice by RNA interference. Nat Genet 33: 401-406, 2003.

31. Holen T, Amarzguioui M, Wiiger MT, Babaie E and Prydz H: Positional effects of short interfering RNAs targeting the human coagulation trigger tissue factor. Nucl Acids Res 30: 1757-1766, 2002. 